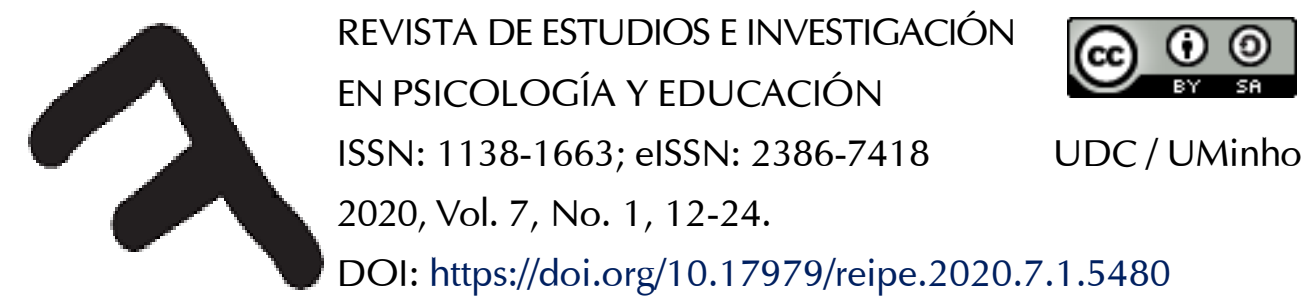

\title{
Questionário de inteligência emocional do professor: adaptação e validação do Emotional Skills and Competence Questionnaire
}

\section{Teacher emotional intelligence questionnaire: adaptation and validation of the Emotional Skills and Competence Questionnaire}

\author{
Sabina Valente (DD*, Abílio Afonso Lourenço (DD** \\ *Univeridade de Évora, **Universidade do Minho
}

\begin{abstract}
Resumo
A inteligência emocional tem motivado um interesse progressivo no domínio da educação. No entanto, não tem sido suficientemente investigada em professores. Como tal, este estudo teve por objetivo adaptar e validar o Emotional Skills and Competence Questionnaire (ESCQ) para professores portugueses. O ESCQ constitui um instrumento de autorrelato da inteligência emocional, tendo por base o modelo teórico de Mayer e Salovey (1997). A amostra foi constituída por 958 professores dos ensinos básicos e secundário. Foram administrados os seguintes questionários: Questionário de Inteligência Emocional do Professor (QIEP), e o Rahim Organizational Conflict Inventory-II- Portuguese Version in School Context (ROCI-IIPViSC). A análise estatística incluiu a técnica de modelação de equações estruturais, tendo por suporte o SPSS/AMOS 25. Desta adaptação e validação resultou o QIEP, um instrumento de avaliação da inteligência emocional para professores. Os resultados da análise fatorial confirmatória demonstram o ajustamento da estrutura fatorial original. O QIEP apresentou um coeficiente de confiabilidade robusto e as diversas características psicométricas evidenciaram a adequada validade e fiabilidade do instrumento. Em conclusão, os resultados deste estudo fornecem evidências de que o QIEP pode ser usado como instrumento para avaliar a inteligência emocional dos professores, no contexto português, podendo ser usado em futuros estudos e práticas educativas.
\end{abstract}

Palavras-chave: inteligência emocional; professores; QIEP; análise fatorial confirmatoria

\begin{abstract}
Emotional intelligence is a growing area of interest in education. Up to now, however, little research attention has been paid to the question of teacher emotional intelligence, probably owing to the lack of assessment tools. The aim of the study reported here was to adapt and validate the Emotional Skills and Competence Questionnaire (ESCQ) for Portuguese teachers. ESCQ is a self-report instrument for measuring emotional intelligence, based on the theoretical model of Mayer and Salovey (1997). A sample of 958 school teachers was supplied with the following survey instruments: the Emotional Skills and Competence Questionnaire for Teachers (ESCQ-T), and the Rahim Organizational Conflict Inventory-II -
\end{abstract}

Sabina Valente (Dorcid.org/0000-0003-2314-3744: Centro de Investigação em Educação e Psicologia (CIEP), Universidade de Évora. Colégio Pedro da Fonseca. Apartado 94. 7002-554 Évora.

Abílio Afonso Lourenço (Dorcid.org/0000-0001-6920-0412: GICAD - Grupo de Investigação sobre Cognição, Aprendizagem e Desempenho, Instituto de Educação, Universidade do Minho. Campus de Gualtar, 4710-057 Braga, Portugal.

Correspondência relativa a este artigo: Sabina Valente - sabinavalentte@gmail.com 
Portuguese Version in School Context (ROCI-II-PViSC). The statistical analysis included structural equation modelling using SPSS/AMOS 25 to adapt and validate the ESCQ-T, an instrument for assessing teacher emotional intelligence. The results of the confirmatory factor analysis showed a good fit with the original factor structure. The ESCQ-T also showed a robust reliability coefficient and good psychometric validity and reliability. The study demonstrates that the Portuguese version of the ESCQ-T is a valid instrument for assessing teacher emotional intelligence and may be used in future studies and in relation to educational practice.

Keywords: emotional intelligence; teachers; ESCQ-T; confirmatory factorial analysis

Nos últimos anos, um grande número de estudos evidencia que a inteligência emocional (IE) é um importante constructo vinculado à atividade profissional dos professores. Diversos estudos demonstram a sua relação positiva com componentes intra e interpessoais, de saúde e profissionais, nomeadamente, no que concerne aos benefícios no desempenho profissional (Cejudo \& López-Delgado, 2017; Valente et al., 2020a), no processo de ensino e aprendizagem (Allen et al., 2014; Yin et al., 2013), na satisfação laboral (Cejudo \& López-Delgado, 2017), no stress e burnout (Subalakshmi, Sunderaraj, \& Manikandan, 2019; Ju et al., 2015), e na gestão da disciplina (Valente et al., 2020b) e do conflito em sala de aula (Valente \& Lourenço, 2020).

Devido aos benefícios que oferece, e como a IE é um constructo em que é possível intervir (Bisquerra, 2000), fica claro que é necessário desenvolver e interceder com programas e instrumentos de avaliação da IE (Extremera et al., 2004) ou, na sua ausência, adaptar aqueles que existem para os professores.

Contudo, apesar do constante aumento de estudos que englobam a IE, uma das limitações é a sua inconsistência teórica (Pérez-González, Petrides, \& Furnham, 2007) e, consequentemente, o surgimento de diferentes instrumentos de medida.

Diversos métodos foram desenvolvidos com o consenso geral de que existem três modelos principais de instrumentos que avaliam a IE (Miao, Humphrey, \& Qian, 2017): (a) Modelos de desempenho: medidas de capacidade da IE com base no desempenho, nos quais a IE individual é avaliada através da realização de várias tarefas e itens de solução de problemas emocionais para os quais existem respostas corretas versus incorretas usando especialistas ou critérios de consenso predeterminados; (b) Modelos de traços de personalidade: baseados nos traços de personalidade ou descritos como modelos mistos, identificam a IE como traço (Petrides \& Furnham, 2001). São instrumentos mistos de IE de autorrelato (e.g., Bar-On, 1997; Goleman, 1995), nos quais aos indivíduos são apresentadas declarações descritivas que combinam uma ampla gama de autoperceções, habilidades sociais, traços e comportamentos relacionados com emoções, onde indicam a extensão em que concordam ou discordam com os itens; e (c) Modelos de capacidades cognitivas: definem e medem a IE como uma capacidade (Petrides \& Furnham, 2001), i.e. são testes de autorrelato de capacidade da IE nos quais aos participantes são apresentadas afirmações descritivas consistentes com a definição de IE de Mayer e Salovey (1997), do seu modelo teórico das capacidades da IE, onde os participantes indicam até que ponto concordam ou discordam com cada um dos itens.

Mayer e Salovey (1997), definem no modelo teórico a IE como "a capacidade para sentir e avaliar a emoção com exatidão, a capacidade para identificar e/ou criar sentimentos quando estes facilitam o pensamento, a capacidade para compreender a emoção e o conhecimento emocional, e a capacidade para regular as emoções que incentivam o crescimento emocional e intelectual" (p. 13). Assim sendo, a IE é um tipo de inteligência que processa informação do foro emocional. O modelo de Mayer e Salovey (1997) tem sido indicado como o mais eficaz para avaliar e compreender a IE, quer em termos de definição e métodos de medida (Extremera \& Fernández-Berrocal, 2005), quer por a sua teoria não ter por base promessas insustentáveis em relação ao potencial da IE (Woyciekoski \& Hutz, 2009).

Considerando o modelo teórico das capacidades da IE, de Mayer e Salovey (1997), foram desenvolvidos distintos instrumentos para avaliar a IE e, em Portugal, foram realizados estudos de adaptação dos seguintes instrumentos (Teques, et al., 2015): (a) Trait Meta-Mood Scale (TMMS; Salovey et al., 1995) adaptado por 
Queirós et al. (2005) à população portuguesa, numa versão modificada, composta por apenas três dimensões, não incluindo as quatro do modelo de base; (b) Multi-factor Emotional Intelligence Scale (MEIS; Mayer, Salovey, \& Caruso, 2002), adaptada por Franco (2003) apenas junto a uma população de professores do $1^{\circ}$ ciclo; e (c) Emotional Skills and Competence Questionnaire (ESCQ; Takšić, 2000), adaptado por Lima-Santos e Faria (2005) a estudantes portugueses, tendo por base o modelo de Mayer e Salovey (1997). De salientar também o Questionário de Autopercepção de Inteligência Emocional (QIE-AP; Teques, 2015), construído e validado na população portuguesa, com base no modelo teórico de Mayer e Salovey (1997) para avaliar a autopercepção das capacidades da IE.

Como Franco (2007) indica, parece existir ainda muito para desenvolver a nível de instrumentos de desempenho da IE em Portugal. Neste sentido, no presente estudo foi usado o ESCQ para adaptação e posterior validação em professores, por ser considerado um importante instrumento de autorrelato da IE (Takšić, 2000), e proporcionar informações relativas ao constructo da IE e às distintas capacidades que o englobam, nomeadamente: a capacidade para perceber e compreender emoções; a capacidade para expressar e classificar emoções; e a capacidade para gerir e regular emoções (Takšić, Mohoric, \& Mirjana, 2009).

Investigações em distintos países confirmaram as potencialidades do ESCQ e, apesar das preocupações com a validade discriminante das medidas de autorrelato (Conte, 2005), existem evidências de que o ESCQ é uma medida confiável e válida que evidencia convergência, e validade preditiva em distintas culturas (Faria et al., 2006). Como anteriormente referido, em Portugal, o ESCQ foi adaptado e validado em alunos dos ensinos secundário e universitário, por Lima-Santos e Faria (2005). Os autores denominaram o instrumento por Questionário de Competência Emocional (QCE) e provaram ser uma medida capaz de avaliar a IE, com resultados a exibir boas qualidades psicométricas.

Sendo a IE definida como a capacidade de perceber, usar, conhecer e regular emoções para facilitar o pensamento (Mayer \& Salovey, 1997) torna-se coerente contrastá-la com a gestão de conflitos. A IE e a gestão de conflito são constructos intrinsecamente relacionados (Hopkins \& Yonker, 2015), permitindo a IE resolver problemas usando tanto a capacidade emocional como a cognitiva (Mayer et al., 2016), e constituindo uma importante variável na gestão do conflito (Chan, Sit, \& Lau 2014; Valente, 2019).

A IE está associada às estratégias usadas para gerir o conflito, sendo esta relação estatisticamente significativa (Valente \& Lourenço, 2020; Villamediana, Donado, \& Zerpa, 2015). Relativamente às capacidades da IE, Villamediana et al. (2015) confirmam que as capacidades para perceber emoções e para gerir emoções explicam o uso das estratégias de integração e compromisso, i.e. quem pontua mais nestas dimensões, apresenta mais tendência a utilizar as estratégias integração e compromisso e menos a estratégia anuência. O estudo de Jordan e Troth (2004) indica uma correlação positiva entre a dimensão da perceção das emoções com o uso da estratégia dominação. Outros estudos demonstram que a capacidade para expressar e compreender emoções pontua mais na estratégia evitação (Godse \& Thingujam, 2010; Jordan \& Troth; 2009). Relativamente à capacidade para gerir e regular emoções, Jordan e Troth (2004) verificam uma correlação positiva entre esta capacidade e as estratégias de integração e dominação. Pelo exposto, é coerente esperar uma associação entre estas variáveis no presente estudo.

Na sequência do ESCQ ser um instrumento multidimensional capaz de medir a IE e com boas qualidades psicométricas (Takšić et al., 2009), o objetivo do presente estudo foi adaptar e validar o ESCQ, em professores dos ensinos básicos e secundário, sendo designado por Questionário de Inteligência Emocional do Professor (QIEP).

\section{Método}

\section{Participantes}

A amostra utilizada foi de conveniência e constituída por 958 professores, dos ensinos básicos e secundário ( $5^{\circ}$ ao $12^{\circ}$ ano de escolaridade), de escolas públicas do norte e centro de Portugal, sendo $61.6 \%$ do género feminino. No que concerne à formação académica, $74.7 \%$ tinham licenciatura, $21.5 \%$ mestrado, $2.2 \%$ 
bacharelato, e $1.6 \%$ doutoramento. Quanto ao tempo de serviço, $11.4 \%$ apresentavam menos de 10 anos de serviço; $26.4 \%$ entre 10-20 anos; $42.7 \%$ entre 21-30 anos; e $19.5 \%$ mais de 30 anos de serviço.

\section{Instrumentos}

Questionário de Inteligência Emocional do Professor (QIEP): Adaptação e validação do ESCQ (Taksic', 2000), para professores dos ensinos básicos e secundário. Instrumento composto por 45 itens, sendo as respostas obtidas através de uma escala de tipo Likert com 6 opções, de 1 (nunca) a 6 (sempre). Apresenta três subescalas: Perceber e compreender emoções; Expressar e classificar emoções; e Gerir e regular emoções.

Rahim Organizational Conflict Inventory II - Portuguese Version in School Context (ROCI-II-PViSC; Valente, Monteiro \& Lourenço, 2017): Instrumento constituído por 28 itens relativos às estratégias adotadas perante o conflito, em sala de aula. As respostas foram avaliadas numa escala tipo Likert de 5 pontos, de 1 (discordo totalmente) a 5 (concordo totalmente). O instrumento inclui cinco subescalas: Integração; Anuência; Dominação; Evitação; e Compromisso.

\section{Procedimentos}

A adaptação e validação do QIEP incluíram os seguintes procedimentos: processo de tradução; adaptação do instrumento; aplicação do pré-teste; aplicação do instrumento; e a sua validação.

\section{Processo de tradução}

Para a utilização do instrumento foi solicitada autorização ao autor da versão original do ESCQ. Posteriormente, o instrumento foi traduzido para português a partir do original, cujo objetivo foi obter uma tradução que conservasse, por um lado, a mesma paridade semântica e psicológica dos itens e, por outro, respeitasse as especificidades gramaticais da língua portuguesa, para atestar a clareza dos itens e o seu fácil entendimento. A tradução foi revista por um professor especialista e nativo da língua inglesa. Foi ainda realizada uma retrotradução para garantir a precisão da tradução linguística.

\section{Adaptação do instrumento}

$\mathrm{Na}$ adaptação do ESCQ, para professores dos ensinos básicos e secundário, apenas foi reformulada a adaptação de alguns itens, tendo-se mantido o número de itens em cada subescala. Deste modo, o QIEP resultante desta adaptação, e posterior validação, é composto por 45 itens e apresenta três subescalas: (a) Perceber e compreender emoções, 15 itens, (e.g., "Quando vejo como um aluno se sente, geralmente sei o que lhe aconteceu"); (b) Expressar e classificar emoções, 14 itens, (e.g., "Consigo expressar os meus sentimentos e emoções por palavras"); e (c) Gerir e regular emoções, 16 itens, (e.g., "Consigo manter-me de bom humor, mesmo quando algo desagradável acontece durante a aula").

\section{Aplicação do pré-teste}

Após a adaptação dos itens foi feita a aplicação do pré-teste e da reflexão falada num grupo de 10 professores, com o objetivo de registar apreciações sobre a interpretação dos itens. Esta reflexão permitiu um melhor ajuste dos itens à amostra em estudo, quer a nível de conteúdo semântico, quer da interpretação e perceção dada a cada um dos itens.

\section{Aplicação do QIEP}

Para a aplicação do instrumento foi solicitada autorização à Direção-Geral de Educação, através da plataforma do Sistema de Monitorização de Inquéritos em Meio Escolar. Posteriormente obteve-se a autorização dos diretores dos agrupamentos de escolas que participaram neste estudo. Seguidamente, o QIEP foi aplicado aos professores em contexto de reunião com os investigadores, na qual foram informados sobre o objetivo do presente estudo. Foi ainda utilizada uma Ficha de Dados Pessoais e Profissionais para recolher informações sobre o género, o tempo de serviço e a formação académica dos participantes. Foram garantidos todos os procedimentos éticos, nomeadamente o anonimato, a confidencialidade das respostas e a voluntariedade na participação. O QIEP (em Anexo) foi aplicado ao longo do ano letivo de 2018/2019. 


\section{Método de análise de dados}

Relativamente ao tratamento estatístico dos dados, foram eliminados todos os casos com missing values e foi usado o método da estimação maximum likelihood no programa AMOS. Foi opção, também, manter os outliers moderados, uma vez que a estatística descritiva da amostra se apresentou ainda adequada. Não foram considerados os índices de modificação para não tornar o modelo mais complexo.

Para examinar a confiabilidade fatorial do QIEP foram usados os índices $\alpha$ de Cronbach, os valores da esfericidade de Bartlett, e o índice de Keiser-Meyer-Olkin (KMO) sendo considerados valores de alpha entre .70 e .90, para uma boa consistência interna da escala (Streiner, 2003). Para confirmar a estrutura fatorial original do QIEP, foi usada uma análise fatorial confirmatória (AFC) com o SPSS/AMOS 25, utilizando a técnica de modelação de equações estruturais (MEE; Lowe, Winzar, \& Ward, 2007). O ajuste do modelo foi avaliado utilizando os seguintes critérios: $\chi^{2}, \chi^{2} / \mathrm{gl}$, Goodness-of-Fit Index (GFI $\geq .90$ ), Adjusted Goodness-ofFit Index (AGFI $\geq .90$ ), Comparative Fit Index (CFI $\geq .95 ; \mathrm{Hu} \&$ Bentler, 1999), Tucker-Lewis Index (TLI $\geq$ 0.95; Hair et al., 2005), Root Mean Square Error of Aproximation (RMSEA < .05; Byrne, 2010), e o Critical $\mathrm{N}$ (CN >200; Hoelter, 1983) indicando que o valor representa adequadamente o tamanho da amostra.

Foi assumido como critério que valores de assimetria maiores que dois e valores de curtose maiores que sete não deveriam ser considerados (Finney \& DiStefano, 2013). Para o coeficiente $r$ de Pearson, assume-se que: (a) $r<.200$ indica um valor muito baixo; (b) entre .200-.399 baixo; (c) entre .400-.699 moderado; (d) entre .700-.899 alto; e (e) entre .900-1 muito alto (Marôco, 2018).

Finalmente, para se testar e comprovar a funcionalidade e operacionalização do QIEP com a gestão de conflitos (ROCI-II-PViSC), foram considerados os valores dos coeficientes de correlação de Pearson existentes entre estes dois constructos, tendo como referência o indicado por Marôco (2018).

\section{Resultados}

A Tabela 1 mostra os dados descritivos (média, desvio padrão, assimetria e curtose) para as variáveis incluídas no modelo.

\section{Tabela 1}

Estatística descritiva correspondente às variáveis incluídas no modelo QIEP

\begin{tabular}{|c|c|c|c|c|c|c|c|c|c|c|}
\hline Dimensão & Itens & $M$ & $D P$ & $A$ & $C$ & Ítens & $M$ & $D P$ & $A$ & $C$ \\
\hline \multirow{8}{*}{$\begin{array}{l}\text { Perceber e } \\
\text { Compreender } \\
\text { Emoções } \\
(\mathrm{PCE})\end{array}$} & QIEP-PCE 03 & 4.73 & 1.07 & -0.93 & 1.20 & QIEP-PCE 27 & 4.16 & 1.19 & -0.55 & 0.14 \\
\hline & QIEP-PCE 06 & 4.98 & 1.26 & -0.17 & -0.21 & QIEP-PCE 30 & 4.03 & 1.20 & -0.45 & 0.28 \\
\hline & QIEP-PCE 09 & 4.72 & 1.14 & -1.44 & 2.21 & QIEP-PCE 33 & 4.22 & 1.23 & -0.74 & 0.62 \\
\hline & QIEP-PCE 12 & 4.43 & 1.29 & -1.24 & 1.10 & QIEP-PCE 36 & 4.14 & 1.18 & -0.68 & 0.74 \\
\hline & QIEP-PCE 15 & 4.55 & 1.17 & -0.86 & 0.62 & QIEP-PCE 39 & 4.60 & 0.95 & -1.14 & 2.83 \\
\hline & QIEP-PCE 18 & 4.23 & 1.17 & -0.86 & 0.91 & QIEP-PCE 42 & 4.54 & 0.98 & -1.04 & 1.98 \\
\hline & QIEP-PCE 21 & 4.65 & 1.02 & -1.27 & 2.36 & QIEP-PCE 44 & 4.63 & 1.05 & -0.89 & 1.20 \\
\hline & QIEP-PCE 24 & 4.46 & 1.08 & -0.93 & 1.80 & & & & & \\
\hline \multirow{7}{*}{$\begin{array}{l}\text { Expressar e } \\
\text { Classificar } \\
\text { Emoções } \\
\text { (ECE) }\end{array}$} & QIEP-ECE 02 & 4.49 & 1.27 & -1.16 & 1.21 & QIEP-ECE 23 & 4.73 & 1.02 & -1.14 & 1.84 \\
\hline & QIEP-ECE 05 & 4.32 & 1.08 & -0.59 & 0.27 & QIEP-ECE 26 & 4.62 & 0.95 & -0.50 & 0.31 \\
\hline & QIEP-ECE 08 & 4.42 & 1.24 & -0.76 & 0.22 & QIEP-ECE 29 & 3.92 & 0.99 & -0.41 & 0.49 \\
\hline & QIEP-ECE 11 & 4.61 & 0.93 & -0.49 & 0.66 & QIEP-ECE 32 & 4.55 & 1.12 & -1.12 & 1.76 \\
\hline & QIEP-ECE 14 & 4.31 & 1.06 & -0.51 & 0.30 & QIEP-ECE 35 & 4.40 & 1.02 & -0.69 & 0.98 \\
\hline & QIEP-ECE 17 & 4.50 & 1.07 & -0.94 & 1.10 & QIEP-ECE 38 & 4.57 & 1.03 & -1.23 & 2.02 \\
\hline & QIEP-ECE 20 & 4.83 & 0.94 & -1.39 & 3.52 & QIEP-ECE 41 & 4.52 & 1.29 & -1.23 & 1.02 \\
\hline \multirow{8}{*}{$\begin{array}{l}\text { Gerir e Regular } \\
\text { Emoções } \\
\text { (GRE) }\end{array}$} & QIEP-GRE 01 & 4.16 & 1.23 & -0.87 & 0.47 & QIEP-GRE 25 & 4.36 & 1.66 & -0.95 & -0.30 \\
\hline & QIEP-GRE 04 & 4.26 & 1.33 & -0.91 & 0.59 & QIEP-GRE 28 & 4.18 & 1.11 & -0.83 & 0.78 \\
\hline & QIEP-GRE 07 & 4.64 & 1.11 & -1.07 & 1.47 & QIEP-GRE 31 & 4.08 & 0.99 & -0.62 & 1.23 \\
\hline & QIEP-GRE 10 & 4.64 & 1.11 & -1.17 & 1.81 & QIEP-GRE 34 & 4.57 & 1.05 & -0.95 & 1.33 \\
\hline & QIEP-GRE 13 & 4.06 & 1.49 & -0.67 & -0.45 & QIEP-GRE 37 & 4.35 & 1.11 & -0.48 & 0.06 \\
\hline & QIEP-GRE 16 & 4.51 & 1.11 & -1.02 & 1.46 & QIEP-GRE 40 & 4.69 & 1.25 & -1.34 & 1.73 \\
\hline & QIEP-GRE 19 & 4.51 & 1.05 & -1.19 & 2.11 & QIEP-GRE 43 & 4.66 & 1.08 & -0.96 & 1.29 \\
\hline & QIEP-GRE 22 & 4.32 & 1.31 & -0.93 & 0.28 & QIEP-GRE 45 & 4.60 & 0.97 & -1.15 & 2.41 \\
\hline
\end{tabular}


O modelo do QIEP apresenta os seguintes índices de ajustamento: $\chi^{2}{ }_{(942)}=1854.200 ; p<.001 ; \chi^{2} / \mathrm{gl}=1.968$; $\mathrm{GFI}=.916 ; \mathrm{AGFI}=.908 ; \mathrm{TLI}=.904 ; \mathrm{CFI}=.909 ; \mathrm{RMSEA}=.032(\mathrm{IC}: .030-.032) ; \mathrm{CN}=524(.05)-540(.01)$. Os valores obtidos indicam que o ajustamento global do modelo é robusto facto que confirma este modelo como um descritor adequado da dimensionalidade do QIEP, assim como apresentaram valores adequados e estatisticamente significativos. É possível verificar, ainda, que às dimensões perceber e compreender emoções, expressar e classificar emoções e gerir e regular emoções, correspondem os alfas de .86, .84 e .79, respetivamente, tendo a escala total um valor de .90 , o que mostra uma alta convergência entre os itens que compõem as diferentes dimensões. Os resultados obtidos confirmam a adequação da matriz para poder realizar a análise. Assim, o valor do teste KMO maior que $0.90(\mathrm{KMO}=.936)$ é adequado. Por sua vez, o valor do teste de Bartlett $\left(\chi^{2}{ }_{(990)}=10838.00 ; p<.001\right)$ mostra a existência de uma correlação significativa entre as variáveis.

Pode constatar-se, na Tabela 2 que não existem parâmetros que exibam estimativas inadequadas como variâncias negativas ou erros de estimativa maiores que um. Por outro lado, também se verifica a ausência de desvios-padrão demasiado elevados ou pequenos indicadores de que os parâmetros respetivos não podem ser estimados com precisão.

\section{Tabela 2}

Estimativas do QIEP: valores não estandardizados, valores estandardizados e erro de estimativa

\begin{tabular}{|c|c|c|c|c|c|c|c|c|}
\hline Dimensões & Itens & $V n E$ & $V E$ & $E E$ & Itens & $V n E$ & $V E$ & $E E$ \\
\hline \multirow{8}{*}{$\begin{array}{l}\text { Perceber e } \\
\text { Compreender } \\
\text { Emoções } \\
(\mathrm{PCE})\end{array}$} & QIEP-PCE3 & 1.00 & --- & --- & QIEP-PCE27 * & 1.57 & 0.59 & 0.14 \\
\hline & QIEP-PCE6 * & 1.18 & 0.42 & 0.17 & QIEP-PCE30 * & 1.52 & 0.57 & 0.14 \\
\hline & QIEP-PCE9 * & 1.36 & 0.54 & 0.13 & QIEP-PCE33 * & 1.59 & 0.58 & 0.14 \\
\hline & QIEP-PCE12 * & 1.27 & 0.44 & 0.13 & QIEP-PCE36 * & 1.56 & 0.59 & 0.14 \\
\hline & QIEP-PCE15 * & 1.18 & 0.45 & 0.12 & QIEP-PCE39 * & 1.42 & 0.67 & 0.12 \\
\hline & QIEP-PCE18 * & 1.71 & 0.66 & 0.14 & QIEP-PCE42 * & 1.44 & 0.66 & 0.12 \\
\hline & QIEP-PCE21 * & 1.49 & 0.66 & 0.13 & QIEP-PCE44 * & 0.93 & 0.40 & 0.10 \\
\hline & QIEP-PCE24 * & 1.45 & 0.60 & 0.13 & & & & \\
\hline \multirow{7}{*}{$\begin{array}{l}\text { Expressar e } \\
\text { Classificar } \\
\text { Emoções (ECE) }\end{array}$} & QIEP-ECE 2 & 1.00 & --- & --- & QIEP-ECE $23 *$ & 1.08 & 0.57 & 0.10 \\
\hline & QIEP-ECE $5 *$ & 0.32 & 0.16 & 0.07 & QIEP-ECE $26 *$ & 0.89 & 0.50 & 0.09 \\
\hline & QIEP-ECE $8 *$ & 1.27 & 0.55 & 0.12 & QIEP-ECE $29 *$ & 0.91 & 0.49 & 0.09 \\
\hline & QIEP-ECE $11 *$ & 1.01 & 0.58 & 0.09 & QIEP-ECE $32 *$ & 0.87 & 0.41 & 0.09 \\
\hline & QIEP-ECE $14 *$ & 1.21 & 0.61 & 0.11 & QIEP-ECE $35 *$ & 0.98 & 0.51 & 0.09 \\
\hline & QIEP-ECE $17 *$ & 1.47 & 0.74 & 0.12 & QIEP-ECE $38 *$ & 1.28 & 0.67 & 0.11 \\
\hline & QIEP-ECE $20 *$ & 1.17 & 0.67 & 0.10 & QIEP-ECE $41 *$ & 1.17 & 0.48 & 0.11 \\
\hline \multirow{8}{*}{$\begin{array}{l}\text { Gerir e Regular } \\
\text { Emoções } \\
\text { (GRE) }\end{array}$} & QIEP-GRE 1 & 1.00 & --- & --- & QIEP-GRE 25* & 0.75 & 0.25 & 0.12 \\
\hline & QIEP-GRE $4 *$ & 0.80 & 0.34 & 0.10 & QIEP-GRE $28 *$ & 0.93 & 0.47 & 0.09 \\
\hline & QIEP-GRE $7 *$ & 0.75 & 0.38 & 0.08 & QIEP-GRE $31 *$ & 0.89 & 0.50 & 0.08 \\
\hline & QIEP-GRE $10 *$ & 0.70 & 0.35 & 0.08 & QIEP-GRE 34 * & 1.11 & 0.59 & 0.10 \\
\hline & QIEP-GRE $13 *$ & 0.87 & 0.33 & 0.11 & QIEP-GRE 37 * & 1.00 & 0.50 & 0.09 \\
\hline & QIEP-GRE $16 *$ & 1.02 & 0.52 & 0.09 & QIEP-GRE $40 *$ & 1.14 & 0.51 & 0.11 \\
\hline & QIEP-GRE $19 *$ & 1.05 & 0.56 & 0.09 & QIEP-GRE $43 *$ & 1.14 & 0.59 & 0.10 \\
\hline & QIEP-GRE $22 *$ & 1.01 & 0.43 & 0.10 & QIEP-GRE $45 *$ & 0.90 & 0.52 & 0.08 \\
\hline \multirow{3}{*}{ Covariâncias } & $\mathrm{PCE} \leftrightarrow \mathrm{ECE} *$ & 0.12 & 0.48 & 0.02 & & & & \\
\hline & $\mathrm{PCE} \leftrightarrow \mathrm{GRE} *$ & 0.12 & 0.49 & 0.02 & & & & \\
\hline & $\mathrm{GRE} \leftrightarrow \mathrm{ECE} *$ & 0.19 & 0.63 & 0.02 & & & & \\
\hline
\end{tabular}

Nota $:$ VNE = Valores não estandardizados; VE = Valores estandardizados; EE $=$ Erro de estimativa $* p \leq .001$

Da análise conjunta da Tabela 2 e da Figura 1 verifica-se que o modelo apresentado assenta nos seguintes pressupostos: (a) o QIEP pode ser explicado por três fatores, nomeadamente perceber e compreender emoções; expressar e classificar emoções; e gerir e regular emoções; (b) a carga fatorial exibida por cada um dos itens está associada apenas ao fator que é suposto medir (carga fatorial alvo) e tem um nível de significância ( $p<$ .001) em todos os itens; (c) não existe correlação entre os erros de estimativa associados a cada um dos itens; e (d) de acordo com o racional teórico no qual está baseado o instrumento, os três fatores estão correlacionados, com valores estatisticamente significativos entre si $(p<.001)$. 


\section{Figura 1}

Modelo QIEP $(n=958)$

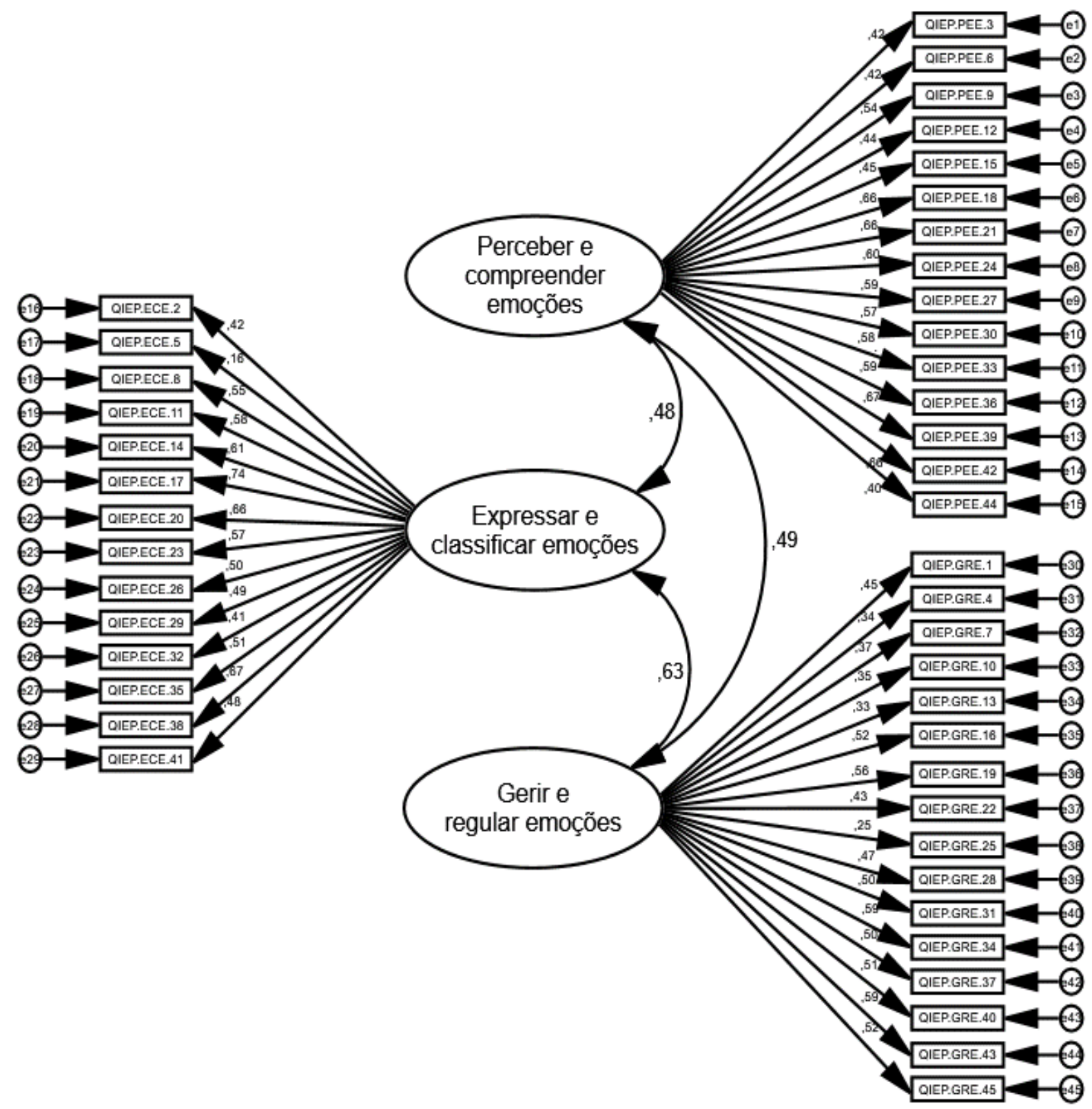

Relativamente às correlações $r$ de Pearson obtidas, os valores indicaram a existência de associações moderadas, positivas e estatisticamente significativas entre as três dimensões que constituem o QIEP. A associação mais consistente verificou-se entre a capacidade de expressar e classificar emoções e a capacidade para gerir e regular emoções $(r=.501 ; p<.01)$. Foi possível observar, igualmente, uma associação entre a capacidade de perceber e compreender emoções com a capacidade de gerir e regular emoções $(r=.414 ; p<.01)$ e com a capacidade de expressar e classificar emoções $(r=.403 ; p<.01)$. Embora com valores fracos, é de salientar que em cada dimensão se registaram associações positivas e estatisticamente significativas entre todos os itens que as constituem, atestando, assim, a homogeneidade e coesão das dimensões que compõem o QIEP.

Por fim, em relação à evidência de validade baseada no relacionamento com outras variáveis, o QIEP foi associado a uma medida de gestão de conflito. Assim, as capacidades da IE para perceber e compreender emoções; expressar e classificar emoções; e gerir e regular emoções mostraram associações significativas com as cinco dimensões do ROCI-II-PViSC (c.f. Tabela 3). 
Tabela 3

Relação entre o QIEP e uma medida de gestão de conflito

\begin{tabular}{lccccc}
\hline & Integração & Anuência & Compromisso & Dominação & Evitação \\
\hline Perceber e compreender emoçõos & $.140^{* * *}$ & $-.111^{* *}$ & $.130^{* *}$ & .032 & -.059 \\
Expressar e classificar emoções & $.145^{* *}$ & $-.074^{*}$ & $.108^{* *}$ & .005 & .051 \\
Gerir e regular emoções & $.118^{* *}$ & $-.127^{* *}$ & $.076^{*}$ & $.074^{*}$ & $.066^{*}$ \\
\hline
\end{tabular}

$* p<.05 ; * * p<.01$

\section{Discussão}

O presente estudo teve por objetivo a adaptação e validação do ESCQ em professores dos ensinos básicos e secundário, de Portugal. Após adaptação e validação o instrumento foi denominado por QIEP. Nesta validação foi testada que a versão portuguesa, em professores, se adequa à estrutura fatorial original, tendo-se efetuado estudos de validade, fiabilidade e de sensibilidade ao nível dos itens e das respetivas dimensões. Os diversos indicadores psicométricos demonstraram a validade deste instrumento. As três dimensões apresentam uma adequada consistência interna $(\alpha=.90)$, sendo próximos dos valores obtidos na versão croata original $(\alpha$ $>$.80; Takšić, 2000), e em estudos posteriores (Faria et al., 2006; Faria \& Lima-Santos, 2012; Takšić et al.,2009). Foram encontrados, ainda, valores de confiabilidade adequados para a esfericidade de Bartlett e para o índice KMO, o que representa a existência de uma correlação significativa entre as variáveis.

De realçar que foram considerados valores de alpha entre .70 e .90 , para uma boa consistência interna do instrumento (Streiner, 2003), verificando-se deste modo que o QIEP apresenta bons valores psicométricos. Ao considerar os respetivos racionais teóricos em que se baseia, os índices de ajustamento global e os parâmetros estimados, poder-se-á concluir que o QIEP se ajusta aos resultados da amostra e confirma o objetivo apresentado para este estudo.

Nesta sequência, os valores de alpha sugerem que os itens são consistentes com as dimensões a que pertencem, e todos os itens estão relacionados com cada uma das suas dimensões na mensuração do respetivo constructo. As correlações entre as três dimensões são significativas, indicando que não são independentes entre si e que convergem na avaliação do constructo da IE. Assim, os resultados obtidos na validação foram consistentes com a estrutura fatorial e conceptual proposta no estudo inicial de Takšić (2000) e suportam a validade de constructo deste instrumento.

Os resultados do presente estudo confirmam a relação entre as capacidades da IE e as estratégias usadas para gerir o conflito, como em estudos anteriores (Godse \& Thingujam, 2010; Jordan \& Troth, 2004; Villamediana et al., 2015). As correlações de Pearson ( $r$ ) revelam que, a maioria das dimensões dos dois constructos, estão associadas entre si, embora fracas, mas estatisticamente significativas, cujos valores das associações variam entre .066 e .145 (Marôco, 2018). De realçar que a dimensão gerir e regular emoções é a única que está associada a todas as estratégias de gestão de conflito e corroboram alguns resultados encontrados noutras investigações (Jordan \& Troth, 2004; Villamediana et al., 2015). De referir, ainda, que as estratégias integração, compromisso e anuência são as únicas que estão associadas às três dimensões da IE.

Quanto às limitações, menciona-se não ter sido realizada uma análise de invariância de medida entre homens e mulheres, apesar de a maioria dos estudos com o ESCQ concluírem que o género feminino tende a apresentar níveis superiores de IE (Faria et al., 2006; Takšić, Mohorić, \& Munjas, 2006). Por outro lado, o presente estudo baseia-se em procedimentos de amostragem não probabilísticos unicamente com professores dos ensinos básicos e secundários. Neste sentido, a generalização dos resultados para outros de professores (e.g., educadores, professores primários, e professores do ensino superior) deverá ser foco de estudo em próximas investigações.

Em suma, o QIEP demonstrou adequadas propriedades psicométricas, contribuindo assim para a existência de uma medida de avaliação da IE dos professores dos ensinos básicos e secundário, em contexto português. Para além de contribuir para a investigação do domínio específico, da IE, poderá ser útil no entendimento das 
distintas capacidades emocionais que o constituem. Neste âmbito, estudos futuros deverão considerar desenhos longitudinais que permitam avaliar a estabilidade e invariância temporal dos resultados do QIEP, relacionandoo com outras variáveis, e investigar a invariância da estrutura fatorial em diferentes culturas.

\section{Referências}

Allen, V. D., MacCann, C., Matthews, G., \& Roberts, R. D. (2014). Emotional Intelligence in Education: From Pop to Emerging Science. In R. Pekrun \& L. Linnenbrink-Garcia (Eds.), International Handbook of Emotions in Education (pp. 162-182). New York and London: Routledge. Recuperado de: https://www.researchgate.net/publication/290162471_Emotional_intelligence_in_education_From_pop_t o_emerging_science

Arbuckle, J. L. (2012). AMOS Version 6. Small waters Corporation.

Bar-On, R. (1997). Bar-On Emotional Quotient Inventory: A measure of emotional intelligence. Toronto: Multi-Health Systems.

Bisquerra, R. (2000). Educación emocional y bienestar. Barcelona: Praxis-Wolters Kluwer.

Byrne, B. M. (2010). Structural Equation Modeling With AMOS - Basic Concepts, Applications, and Programming (2nd ed.). Mahwah, NJ: Erlbaum.

Cejudo, J., \& López-Delgado, M. L. (2017). Importancia de la inteligencia emocional en la práctica docente: un estudio con maestros. Psicología Educativa 23, 29-36. https://doi.org/10.1016/j.pse.2016.11.001

Chan, J. C. Y., Sit, E. N. M., \& Lau, W. M. (2014). Conflict management styles, emotional intelligence and implicit theories of personality of nursing students: A cross-sectional study. Nurse Education Today 34, 934-939. https://doi.org/10.1016/j.nedt.2013.10.012

Conte, J. M. (2005). A review and critique of emotional intelligence measures. Journal of Organizational Behavior, 26, 433-440. https://doi.org/10.1002/job.319

Extremera, N., Fernández-Berrocal, P, Mestre, J.M., \& Guil, R. (2004). Medidas de evaluación de la inteligencia emocional. Revista Latinoamericana de Psicología, 36(2), 209-228. https://www.redalyc.org/ articulo.oa?id=80536203

Extremera, N., \& Fernández-Berrocal, P. (2005). Perceived emotional intelligence and life satisfaction: Predictive and incremental validity using the Trait Meta-Mood Scale. Personality and Individual Differences, 39, 937-948. https://doi.org/10.1016/j.paid.2005.03.012

Faria, L, \& Lima-Santos, N. (2012). Emotional Intelligence in the Portuguese academic context validation studies of "The emotional skills and competence questionnaire" (ESCQ). Behavioral Psychology/Psicología Conductual, 20(1), 91-102. https://www.behavioralpsycho.com/wp-content/ uploads/2019/08/06.Faria_20-1.pdf

Faria, L., Santos, N. L., Takšić, V., Räty, H., Molander, B., Holmström, S., Jansson, J., Avsec A., Extremera, N., Fernández-Berrocal, P, \& Toyota, H. (2006). Cross-cultural validation of the Emotional Skills and Competence Questionnaire (ESCQ). Psicologia, XX(2), 95-127. https://doi.org/10.17575/rpsicol.v20i2.390

Franco, M. (2003). A gestão das emoções na sala de aula: Projecto de modificação das atitudes de um grupo de docentes do $1^{\circ}$ ciclo do ensino básico. Lisboa: Fundação Calouste Gulbenkian.

Franco, M. (2007). Inteligência emocional: Modelos, instrumentos de avaliação e limites. In A. Cadeias \& L. Almeida (Eds.), Inteligência humana: Investigação e aplicações, Vol. 1 (pp.

73-96). Évora: Quarteto. http://hdl.handle.net/10174/1812

Finney, S., \& DiStefano, C. (2013). Non-normal and categorical data in structural equation models. In G. Hancock and R. Mueller (Eds.), A second course in structural equation modeling (pp. 439-492). Greenwich, CT: Information Age.

Godse, A. S., \& Thingujam, N. S. (2010). Perceived emotional intelligence and conflict resolution styles among information technology professionals: Testing the mediating role of personality. Singapore Management Review, 32(1), 69-83. 
Goleman, D. (1995). Emotional intelligence. New York: Bantam Books. [Tradução: Inteligência Emocional. Lisboa: Temas e Debates, 1995.]

Hoelter, J.W. (1983). The analysis of covariance structures: Goodness-of-fit indices. Sociological Methods and Research, 11, 325-344. https://doi.org/10.1177/0049124183011003003

Hopkins, M., \& Yonker, R. (2015). Managing conflict with emotional intelligence: Abilities that make a difference. Journal of Management Development, 34(2), 226-244. https://doi.org/10.1108/JMD-04-20130051

Jordan, P. J., \& Troth, A. C. (2004). Managing emotions during team problem solving: Emotional intelligence and conflict resolution. Human Performance, 17(2), 195-218. https://doi.org/10.1207/s15327043hup1702_4

Ju, C., Lan, J., Li, Y., Feng, W., \& You, X. (2015). The mediating role of workplace social support on the relationship between trait emotional intelligence and teacher burnout. Teaching and Teacher Education 51, 58-67. https://doi.org/10.1016/j.tate.2015.06.001

Lima-Santos, N., \& Faria, L. (2005). Inteligência emocional: Adaptação do "Emotional Skills and Competence Questionnaire" (ESCQ) ao contexto português. Revista da Faculdade de Ciências Humanas e Sociais da Universidade Fernando Pessoa, 2, 275-289. http://hdl.handle.net/10284/668

Lowe, B., Winzar, H., \& Ward, S. (2007). Essentials of SPSS for Windows versions 14 \& 15: a business approach. South Melbourne, Vic.: Thomson Learning Australia.

Marôco, J. (2018). Análise estatística com o SPSS statistics, (7a edição). Pêro Pinheiro: ReportNumber.

Mayer, J. D., \& Salovey, P. (1997). What is Emotional Intelligence? In P. Salovey \& J. Sluyter (Ed.), Emotional development and emotional intelligence: Educational implications (pp.3-31). New York: Basic Books.

Mayer, J., Salovey, P., \& Caruso, D. (2002). Mayer-Salovey-Caruso Emotional Intelligence Test (MSCEIT) user's manual. Toronto: Multi-Health Systems.

Mayer, J. D., Caruso, D. R., \& Salovey, P. (2016). The ability model of emotional intelligence: Principles and updates. Emotion Review, 8(4), 290-300. https://doi.org/10.1177/1754073916639667

Miao, C., Humphrey, R.H., \& Qian, S. (2017). A meta-analysis of emotional intelligence and work attitudes. Journal of Occupational and Organizational Psychology, 90(2), 177-202. https://doi.org/10.1111/joop.12167

Morrinson, J. (2008). The relationship between emotional intelligence competencies and preferred conflicthandling styles. Journal of Nursing Management, 16(8), 974-983. https://doi.org/10.1111/j.13652834.2008.00876.x

Pérez-González, J., Petrides, K., \& Furnham, A. (2007). La medida de la inteligencia emocional rasgo. In J. Navas \& P. Berrocal (Eds.), Manual de inteligencia emocional (pp. 81-93). Madrid: Pirámide.

Petrides, K., \& Furnham, A. (2001). Trait emotional intelligence: Psychometric investigation with reference to established trait taxonomies. European Journal of Personality, 15(6), 425-448. https://doi.org/10.1002/per.416

Queirós, M., Fernández-Berrocal, P., Extremera, N., Carral, J., \& Queirós, P. (2005). Validação e fiabilidade da versão portuguesa modificada da Trait Meta-Mood Scale. Psicologia,Educação e Cultura, 9(1), 199-216. http://emotional.intelligence.uma.es/pdfs/valid\%20e\%20fiabilidade\%20da\%20vers\%20portuguesa $\% 20 \mathrm{mo}$ dificada\%20da\%20tmms.pdf

Salovey, P., Mayer, D., Goldman, L., Turvey, C., \& Palfai, P. (1995). Emotional attention, clarity, and repair: Exploring emotional intelligence using the Trait Meta-Mood Scale. In J. Pennebaker (Ed.), Emotion, disclosure, \& Health (pp. 125-151). Washington, DC: American Psychological Association.

Streiner, D. L. (2003). Being inconsistent about consistency: When coefficient alpha does and doesn't matter. Journal of Personality Assessment, 80(3), 217-222. https://doi.org/10.1207/S15327752JPA8003_01

Subalakshmi, S., Sunderaraj, R., \& Manikandan, M. (2019). Impact of emotional intelligence on stress: with special reference to government school teachers. Journal of Entrepreneurship and Management, 8(1), 7 21. http://www.publishingindia.com/jem/50/impact-of-emotional-intelligence-on-stress-with-special-refer ence-to-government-school-teachers/781/5426/ 
Takšić, V. (2000). Emotional Skills and Competence Questionnaire. Edição do Autor.

Takšić, V., Mohorić, T., \& Munjas, R. (2006). Emocionalna inteligencija: Teorija, operacionalizacija i povezanost $\mathrm{s}$ pozitivnom psihologijom. [Emotional Intelligence: Theory, Operationalization, Implementation and Relationship with positive Psychology]. Društvena istraživanja, 84-85, 729-752.

Takšić, V., Mohoric, T., \& Mirjana, D. (2009). Emotional skills and competence questionnaire (ESCQ) as a self-report measure of emotional intelligence. Horizons of Psychology, 18(3), 7-21. https://doi.org/10.1037/t63691-000

Teques, A. P., Llorca-Ramón, G., Bueno-Carrera, G., Pais-Ribeiro, J., \& Teques, P. (2015). Desenvolvimento e avaliação das características psicométricas do Questionário de Auto-Percepção de Inteligência Emocional (QIE-AP). Psicologia: Reflexão e Crítica, 28(2), 270-279. https://doi.org/10.1590/1678-7153.201528207

Valente, S. (2019). Influência da inteligência emocional na gestão de conflito na relação professor-aluno(s). Revista de Estudios e Investigación en Psicología y Educación, 6(2), 101-113. https://doi.org/10.17979/ reipe.2019.6.2.5786

Valente, S., Monteiro, A. P., \& Lourenço, A. A. (2017). Adaptação e validação da escala de gestão de conflitos entre professores-alunos. Revista de Estudios e Investigación en Psicología y Educación, Vol. Extr. (2), 4145. https://doi.org/10.17979/reipe.2017.0.02.2512

Valente, S., \& Lourenço, A. A. (2020). Conflict in the classroom: How teachers' emotional intelligence influences conflict management. Frontiers in Education, 5(5). https://doi.org/10.3389/feduc.2020.00005

Valente, S., Veiga-Branco, A., Rebelo, H., Lourenço, A. A., \& Cristóvão, A. M. (2020a). The relationship between emotional intelligence ability and teacher efficacy. Universal Journal of Educational Research, 8(3), 916-923. https://doi.org/10.13189/ujer.2020.080324

Valente, S., Lourenço, A. A., Alves, P., \& Domínguez-Lara, S. (2020b). The role of the teacher's emotional intelligence for efficacy and classroom management. CES Psicología, 13(2), 18-31. http://dx.doi.org/1 0.21615/cesp.13.2.2

Villamediana, J., Donado, A., \& Zerpa, C. E. (2015). Estilos de manejo de conflictos, inteligencia emocional y desarrollo moral. Rev. Dimensión Empresarial 13(1), 73-94. https://doi.org/10.15665/rde.v13i1.339

Woyciekoski, C., \& Hutz, C. (2009). Inteligência emocional: Teoria, pesquisa, medida, aplicações e controvérsias. Psicologia: Reflexão e Críticas, 22(1), 1-11. https://doi.org/10.1590/S0102-79722009000100002

Yin, H., Lee, J. C. K., Zhang, Z., \& Jin, Y. (2013). Exploring the relationship among teachers' emotional intelligence, emotional labor strategies and teaching satisfaction. Teaching and Teacher Education, 35, 137-145. https://doi.org/10.1016/j.tate.2013.06.006

Fecha de recepción: 5 de julio de 2019.

Fecha de revisión: 21 de abril de 2020.

Fecha de aceptación: 25 de abril de 2020.

Fecha de publicación: 1 de julio de 2020. 


\title{
ANEXO
}

\section{Questionário de Inteligência Emocional do Professor (QIEP)}

\author{
Autores: Sabina Valente \& Abílio Afonso Lourenço \\ Adaptado de: Vladimir Takšić (2000)
}

O presente Questionário é anónimo e confidencial.

Por favor, leia cada uma das seguintes afirmações e responda colocando um círculo no número correspondente à sua opinião, usando seguinte a escala:

\section{Nunca; 2. Raramente; 3. Poucas vezes; 4. Algumas vezes; 5. Frequentemente; 6. Sempre}

\begin{tabular}{|c|c|}
\hline 1 & $\begin{array}{l}\text { Consigo manter-me de bom humor, mesmo quando algo desagradável acontece durante } \\
\text { a aula. }\end{array}$ \\
\hline 2 & Consigo exprimir os meus sentimentos e emoções por palavras. \\
\hline 3 & Quando encontro um aluno, apercebo-me logo da sua disposição. \\
\hline 4 & $\begin{array}{l}\text { Consigo manter-me de bom humor, mesmo quando os meus alunos estão de mau } \\
\text { humor. }\end{array}$ \\
\hline 5 & No decurso da aula, quando algo me desagrada demonstro-o de imediato. \\
\hline 6 & Quando vejo como um aluno se sente, geralmente sei o que lhe aconteceu. \\
\hline 7 & As experiências desagradáveis que ocorrem na aula ensinam-me o que não devo fazer. \\
\hline 8 & Facilmente consigo cativar os meus alunos. \\
\hline 9 & Consigo perceber quando os meus alunos estão tristes ou dececionados. \\
\hline 10 & Quando um aluno me elogia, trabalho com maior entusiasmo. \\
\hline 11 & Consigo facilmente descrever as emoções que sinto. \\
\hline 12 & Consigo facilmente ver as mudanças de humor nos meus alunos. \\
\hline 13 & Quando não gosto de alguém mostro o que sinto. \\
\hline 14 & Exprimo bem as minhas emoções durante as aulas. \\
\hline 15 & Consigo facilmente animar um aluno quando este se encontra triste. \\
\hline 16 & Quando estou de bom humor é difícil ficar mal disposto(a). \\
\hline 17 & Consigo facilmente expressar o que sinto. \\
\hline 18 & Ao observar um aluno junto de outros, sou capaz de descrever bem as suas emoções. \\
\hline 19 & $\begin{array}{l}\text { Quando estou de bom humor, todos os problemas que surgem durante a aula parecem } \\
\text { ter solução. }\end{array}$ \\
\hline 20 & Consigo descrever o meu estado emocional atual. \\
\hline 21 & Consigo perceber quando um aluno se sente desanimado. \\
\hline 22 & Quando estou com os meus alunos, tenho cuidado com a forma como me comporto. \\
\hline 23 & Posso afirmar que conheço bem o meu estado emocional. \\
\hline 24 & Consigo descrever os sentimentos de um aluno a partir da sua expressão facial. \\
\hline 25 & Quando estou feliz e bem-humorado(a), trabalho melhor. \\
\hline 26 & O meu comportamento reflete os meus sentimentos mais profundos. \\
\hline 27 & Consigo detetar a inveja disfarçada nos outros. \\
\hline 28 & Se quiser consigo resolver problemas que parecem sem solução. \\
\hline 29 & Os alunos são sempre capazes de descrever o meu estado de humor. \\
\hline 30 & Percebo quando um aluno tenta esconder o seu mau humor. \\
\hline 31 & Consigo facilmente persuadir um aluno de que não há razões para se preocupar. \\
\hline 32 & Por norma, sei as razões porque me sinto mal. \\
\hline 33 & Percebo quando um aluno se sente culpado. \\
\hline
\end{tabular}

\begin{tabular}{|l|l|l|l|l|l|}
\hline 1 & 2 & 3 & 4 & 5 & 6 \\
\hline 1 & 2 & 3 & 4 & 5 & 6 \\
\hline 1 & 2 & 3 & 4 & 5 & 6 \\
\hline 1 & 2 & 3 & 4 & 5 & 6 \\
\hline 1 & 2 & 3 & 4 & 5 & 6 \\
\hline 1 & 2 & 3 & 4 & 5 & 6 \\
\hline 1 & 2 & 3 & 4 & 5 & 6 \\
\hline 1 & 2 & 3 & 4 & 5 & 6 \\
\hline 1 & 2 & 3 & 4 & 5 & 6 \\
\hline 1 & 2 & 3 & 4 & 5 & 6 \\
\hline 1 & 2 & 3 & 4 & 5 & 6 \\
\hline 1 & 2 & 3 & 4 & 5 & 6 \\
\hline 1 & 2 & 3 & 4 & 5 & 6 \\
\hline 1 & 2 & 3 & 4 & 5 & 6 \\
\hline 1 & 2 & 3 & 4 & 5 & 6 \\
\hline 1 & 2 & 3 & 4 & 5 & 6 \\
\hline 1 & 2 & 3 & 4 & 5 & 6 \\
\hline 1 & 2 & 3 & 4 & 5 & 6 \\
\hline 1 & 2 & 3 & 4 & 5 & 6 \\
\hline 1 & & & & & \\
\hline 1 & 2 & 3 & 4 & 5 & 6 \\
\hline 1 & 2 & 3 & 4 & 5 & 6 \\
\hline 1 & 2 & 3 & 4 & 5 & 6 \\
\hline 1 & 2 & 3 & 4 & 5 & 6 \\
\hline 1 & 2 & 3 & 4 & 5 & 6 \\
\hline 1 & 2 & 3 & 4 & 5 & 6 \\
\hline 1 & 2 & 3 & 4 & 5 & 6 \\
\hline 1 & 2 & 3 & 4 & 5 & 6 \\
\hline 1 & 2 & 3 & 4 & 5 & 6 \\
\hline 1 & 2 & 3 & 4 & 5 & 6 \\
\hline 1 & 2 & 3 & 4 & 5 & 6 \\
\hline 1 & 2 & 3 & 4 & 5 & 6 \\
\hline 1 & 2 & 3 & 4 & 5 & 6 \\
\hline
\end{tabular}




\begin{tabular}{|l|l|}
\hline 34 & Durante as aulas procuro moderar as emoções desagradáveis e reforçar as positivas. \\
\hline 35 & Facilmente manifesto carinho aos meus alunos. \\
\hline 36 & Percebo quando um aluno tenta esconder os seus verdadeiros sentimentos. \\
\hline 37 & Habitualmente o que sinto não tem nada de errado. \\
\hline 38 & Consigo nomear e descrever a maioria das minhas emoções. \\
\hline 39 & Percebo quando um aluno está triste. \\
\hline 40 & Cumpro os meus deveres e obrigações com prontidão, em vez de estar a pensar neles. \\
\hline 41 & Consigo reconhecer a maioria dos meus sentimentos. \\
\hline 42 & Percebo quando o comportamento de um aluno varia em função do seu humor. \\
\hline 43 & Tento manter o bom humor durante as aulas. \\
\hline 44 & Sei como surpreender de forma agradável os meus alunos. \\
\hline 45 & Quanto aos meus sentimentos, é normal sentir o que sinto neste momento. \\
\hline
\end{tabular}

\begin{tabular}{|l|l|l|l|l|l|}
\hline 1 & 2 & 3 & 4 & 5 & 6 \\
\hline 1 & 2 & 3 & 4 & 5 & 6 \\
\hline 1 & 2 & 3 & 4 & 5 & 6 \\
\hline 1 & 2 & 3 & 4 & 5 & 6 \\
\hline 1 & 2 & 3 & 4 & 5 & 6 \\
\hline 1 & 2 & 3 & 4 & 5 & 6 \\
\hline 1 & 2 & 3 & 4 & 5 & 6 \\
\hline 1 & 2 & 3 & 4 & 5 & 6 \\
\hline 1 & 2 & 3 & 4 & 5 & 6 \\
\hline 1 & 2 & 3 & 4 & 5 & 6 \\
\hline 1 & 2 & 3 & 4 & 5 & 6 \\
\hline 1 & 2 & 3 & 4 & 5 & 6 \\
\hline
\end{tabular}

OBRIGADA PELA COLABORAÇÃO 